\title{
Optimal Time of Initiating Adjuvant Chemotherapy After Curative Surgery in Colorectal Cancer Patients
}

\author{
Kyu Min Kang, Kyung Sook Hong, Gyoung Tae Noh, Bo-Young Oh, Soon Sup Chung, Ryung-Ah Lee, \\ Kwang Ho Kim \\ Department of Surgery, Ewha Womans University School of Medicine, Seoul, Korea
}

Purpose: Adjuvant chemotherapy is routinely recommended for locally advanced colorectal cancer (CRC). There are very few data for the optimal starting date of adjuvant chemotherapy after the surgery. This study aimed to evaluate the effectiveness of earlier adoption of adjuvant chemotherapy after curative surgery for stage III CRC.

Methods: In this study, 159 patients with stage III CRC, who had undergone a curative resection, were enrolled retrospectively. Patients were categorized into 3 groups representing different timings to initiate the chemotherapy; less than 2 weeks (group 1), 3 to 4 weeks (group 2), and more than 5 weeks (group 3). The overall survival rate (OS) and the relapsefree survival rate (RFS) were analyzed to evaluate the effectiveness of adjuvant chemotherapy.

Results: The 5-year OSs of the patients were $73.7 \%$ in group 1, 67.0\% in group 2, and 55.2\% in group 3. The 5-year RFSs of the patients were $48.8 \%$ in group $1,64.7 \%$ in group 2 , and $57.1 \%$ in group 3 . There were no significant differences in either the OS or the RFS $(\mathrm{P}=0.200, \mathrm{P}=0.405)$.

Conclusion: Starting chemotherapy earlier than 6 weeks after surgery does not show any significant difference. Thus, although adjuvant chemotherapy should preferably begin within 6 weeks, the starting date should not necessarily be hastened, and the patient's general condition should be taken into consideration.

Keywords: Adjuvant chemotherapy; Colorectal neoplasms; Prognosis; Therapeutic guidelines

\section{INTRODUCTION}

Colorectal cancer (CRC) is a major public health problem accounting for over one million cases of new cancers and about half a million deaths annually worldwide [1]. It is the second leading cause of cancer-related death in Western countries and the third most common cancer in Korea [2,3]. While surgical resection remains the cornerstone of treatment for CRC, a significant propor-

Received: April 12, 2013 - Accepted: May 21, 2013

Correspondence to: Kwang Ho Kim, M.D.

Department of Surgery, Ewha Womans University Mokdong Hospital, Ewha Womans University School of Medicine, 1071 Anyangcheon-ro,

Yangcheon-gu, Seoul 158-710, Korea

Tel: +82-2-2650-6100, Fax: +82-2-2644-7984

E-mail: eastgate@ewha.ac.kr

(C) 2013 The Korean Society of Coloproctology

This is an open-access article distributed under the terms of the Creative Commons Attribution NonCommercial License (http://creativecommons.org/licenses/by-nc/3.0) which permits unrestricted noncommercial use, distribution, and reproduction in any medium, provided the original work is properly cited tion of patients has suffered from relapses and deaths even after the surgery. With great efforts to improve postoperative survival, adjuvant chemotherapy, due to its significant survival benefits, has been routinely recommended after a curative surgical resection of stage III CRC [3]. In large, randomized, clinical trials, postoperative adjuvant chemotherapy in stage III CRC provided an approximately one-third reduction in the relative risk of cancer recurrence and cancer-related death [4]. Guidelines published by the American Society of Clinical Oncology and the National Comprehensive Cancer Network for CRC recommend that all patients with stage III disease should undergo surgical resection and receive adjuvant chemotherapy [5]. However, although the use of adjuvant treatment is prevalent, the optimal time to start chemotherapy after surgery in CRC is not well established [6]. It is usually accepted that adjuvant chemotherapy should begin within 8 weeks after surgery, and most clinical trials mandate that it should be started within 6 to 8 weeks after surgery. A large, randomized study conducted with stage III CRC patients in the United States reports that, compared with adjuvant chemotherapy initiated 
within one month after surgery, adjuvant chemotherapy initiated beyond three months after surgery was associated with a 50\% increase in colon-cancer-specific mortality [7]. Other studies report no clinical benefits of chemotherapy when it is delayed beyond 3 months after surgery [8]. Because of such reports, a routine clinical assumption has been that chemotherapy should be commenced as soon as possible following surgery. However, many clinicians have the experience that chemotherapy initiated too soon after surgery leads to more side effects induced by the agents of chemotherapy. These complications may interrupt the treatment agenda of chemotherapy and may even cause death [9]. With this controversy, our study aims to quantitatively evaluate the effectiveness of beginning adjuvant chemotherapy early, within 6 weeks after curative surgery.

\section{METHODS}

From January 2002 to December 2009, 159 patients with stage III CRC who had undergone surgical resection were enrolled in this study. All the patients had been pathologically diagnosed with stage III CRC after elective and curative surgeries, and had received adjuvant chemotherapy. Patients who had taken neoadjuvant chemotherapy and perioperative radiotherapy were excluded. The medical records of patients were reviewed retrospectively. $\mathrm{Pa}$ tients were categorized into three groups representing different times for starting adjuvant chemotherapy after surgery; patients having adjuvant chemotherapy within 2 weeks, 3 to 4 weeks, and 5 to 6 weeks after surgery were categorized as group 1, group 2, and group 3, respectively.

To evaluate the efficacy of adjuvant chemotherapy according to the timing of its initiation, we analyzed and compared the 5-year overall survival rates (OSs) and the relapse-free survival rates (RFSs) after surgery between the groups. The OSs and the RFSs were estimated by using the Kaplan-Meier method, and the differences between the groups were assessed using the log-rank test. Univariate and multivariate Cox proportional hazards models were used to adjust for the imbalances in the conventional prognostic factors. A two-sided P-value of less than 0.05 was considered to represent statistical significance. IBM SPSS ver. 18.0 (IBM Co., Armonk, NY, USA) was used for statistical analyses.

\section{RESULTS}

Of the 159 patients enrolled in this study, 73 patients were male, and 86 patients were female. The mean age of the patients was 63.7 years (range, 28 to 89 years) old. The mean interval from the surgery to the initiation of adjuvant chemotherapy was 22.4 days, with a range of 8 to 122 days. One hundred twenty-one patients had chemotherapy intravenously, and 38 patients had it orally. Among the patients with intravenous chemotherapy, 74 patients had chemotherapy with 5-fluorouracil and leucovorin (the regimen of 5-FU/LV), and 47 patients had oxaliplatin, 5-fluorouracil and leucovorin (the regimen of FOLFOX-4). Of the 38 patients who had oral chemotherapy, 19 patients received Xeloda (capecitabine, Roche, Basel, Switzerland) while 15 patients received Fluridine (doxifluridine, Samjin Pharm., Seoul, Korea) and 4 patients received UFT (tegafur combinations, Jeil pharmaceutical Co., Seoul, Korea) (Table 1).

A total of 72 patients, group 1, received chemotherapy within 2 weeks after surgery. The mean age of those patients was 60.7 years old. The number of males and females was 36 each. The mean follow-up duration was 41.5 months. A total 54 of patients, group 2, received chemotherapy within 3-4 weeks after surgery. The mean age of those patients was 66.1 years old. The numbers of males and females were 25 and 29 , respectively. The mean follow-up duration was 50.3 months. A total of 33 patients, group 3, received chemotherapy within 5-6 weeks after surgery. Of those patients, 12 were male, and 21 were female, and the mean age was 66.3 years old. The mean follow-up duration was 47.4 months. There were no significant differences in either the sex distribution or the mean duration of follow-up between the groups $(\mathrm{P}=0.428$, $\mathrm{P}=0.121)$. However, the mean age of group 1 was significantly younger than those of the other two groups $(\mathrm{p}=0.021)$ (Table 2).

The 5-year OSs were $73.7 \%$ in group $1,67.0 \%$ in group 2 , and $55.2 \%$ in group 3 . There was no statistical significance in the 5 -year OSs between these 3 groups (log-rank $\mathrm{P}=0.200$ ) (Table 3 ). The Kaplan-Meier curves for the 5-year OS are shown in Fig. 1. The results of an analysis with the multivariate Cox proportional hazards model showed that the hazards ratios (HRs) of the overall survival were 0.699 (95\% confidence interval [CI], 0.335 to 1.462 )

Table 1. Demographic characteristics of patients $(n=159)$

\begin{tabular}{lc}
\hline Characteristic & Value \\
\hline Age (yr), mean \pm SD & $63.7 \pm 12.42$ \\
Sex (male:female) & $73: 86$ \\
Interval from surgery to chemotherapy (day), mean \pm SD & $22.4 \pm 17.14$ \\
Regimen of adjuvant chemotherapy & \\
5-Fluorouracil / leucovorin & 74 \\
FOLFOX-4 & 47 \\
Oral agents & 38 \\
\hline
\end{tabular}

SD, standard deviation.

Table 2. Distributions of age and sex in each group

\begin{tabular}{|c|c|c|c|c|}
\hline Variable & $\begin{array}{c}\text { Group } 1 \\
\text { (within } 2 \text { wk) }\end{array}$ & $\begin{array}{l}\text { Group } 2 \\
\text { (3-4 wk) }\end{array}$ & $\begin{array}{l}\text { Group } 3 \\
\text { (5-6 wk) }\end{array}$ & $\begin{array}{l}\text { P-value } \\
(<0.05)\end{array}$ \\
\hline No. of patients & 72 & 54 & 33 & - \\
\hline Age $(y r)$, mean \pm SD & $60.7 \pm 12.14$ & $66.1 \pm 12.16$ & $66.3 \pm 12.38$ & 0.021 \\
\hline Sex (male:female) & $36: 36$ & $25: 29$ & $12: 21$ & 0.428 \\
\hline $\begin{array}{l}\text { Follow-up duration (mo), } \\
\text { mean } \pm \text { SD }\end{array}$ & $41.5 \pm 21.01$ & $50.3 \pm 24.56$ & $47.4 \pm 29.28$ & 0.121 \\
\hline
\end{tabular}


in group 1 and 0.596 (95\% CI, 0.287 to 1.236) in group 2, but this difference was not statistically significant $(\mathrm{P}=0.366)$. The results obtained for age by using the univariate Kaplan-Meier method were statistically significant $(\mathrm{P}=0.017)$. The $\mathrm{HR}$ obtained for age by using the multivariate Cox proportional hazards model was 1.041 (95\% CI, 0.012 to 1.070$)(\mathrm{P}=0.005)$. The sex distribution showed no difference between the three groups based on the univariate analysis; the $\mathrm{HR}$ was 0.886 (95\% CI, 0.485 to 1.620$)(\mathrm{P}=$ 0.695) (Table 3).

The 5-year RFSs were $48.8 \%$ in group 1, 64.7\% in group 2, and $57.1 \%$ in group 3 ( $\log$-rank $\mathrm{P}=0.405$ ) (Table 4$)$. The results of an analysis with the multivariate Cox proportional hazard regression model for the 5-year RFS are shown in Table 4. The HRs of the 5 -year RFS were 0.985 (95\% CI, 0.493 to 1.966) in group 1 and 0.656 (95\% CI, 0.313 to 1.376 ) in group 2. However, there was no statistical significance in the multivariate analysis of the 5-year

Table 3. Univariate Kaplan-Meier method and multivariate Cox proportional hazards model of 5-year overall survival

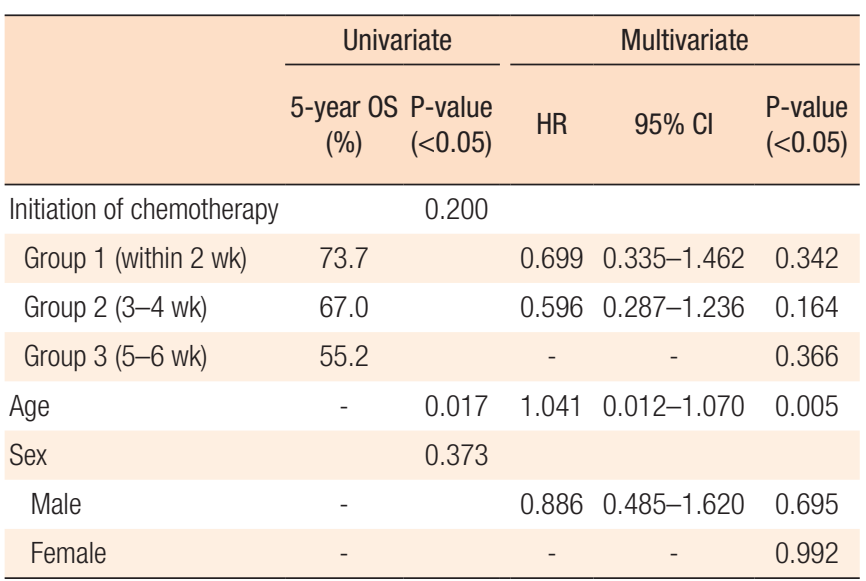

OS, overall survival; HR, hazard ratio; $\mathrm{Cl}$, confidence interval.

Table 4. Univariate Kaplan-Meier method and multivariate Cox proportional hazards model of 5-year relapse-free survival

\begin{tabular}{|c|c|c|c|c|c|}
\hline & \multicolumn{2}{|c|}{ Univariate } & \multicolumn{3}{|c|}{ Multivariate } \\
\hline & $\begin{array}{c}\text { 5-year } \\
\text { RFS } \\
(\%)\end{array}$ & $\begin{array}{l}\text { P-value } \\
(<0.05)\end{array}$ & $H R$ & $95 \% \mathrm{Cl}$ & $\begin{array}{l}\text { P-value } \\
(<0.05)\end{array}$ \\
\hline Initiation of chemotherapy & & 0.405 & & & \\
\hline Group 1 (within 2 wk) & 48.8 & & 0.985 & $0.493-1.966$ & 0.965 \\
\hline Group 2 (3-4 wk) & 64.7 & & 0.656 & $0.313-1.376$ & 0.265 \\
\hline Group 3 (5-6 wk) & 57.1 & & - & - & 0.377 \\
\hline Age & - & 0.000 & 1.005 & $0.983-1.027$ & 0.654 \\
\hline Sex & - & 0.808 & & & \\
\hline Male & - & & 0.944 & $0.556-1.602$ & 0.832 \\
\hline Female & - & & - & - & 0.899 \\
\hline
\end{tabular}

$\mathrm{RFS}$, relapse-free survival; $\mathrm{HR}$, hazard ratio; $\mathrm{Cl}$, confidence interval.
RFS ( $\mathrm{P}=0.377)$ (Table 4). The Kaplan-Meier curves for the 5-year RFS are shown in Fig. 2.

\section{DISCUSSION}

There are several different hypotheses regarding the mechanism behind local recurrence and distant metastasis after radical resection of a tumor. Some studies with animal models suggest that surgery might increase the number of circulating tumor cells and accelerate the growth of metastatic infiltrates. These results are associated with the reduction of angiogenesis inhibitors, such as angiostatin, after removal of the primary tumor [9-12]. Surgery has been shown to enhance production of oncogenic growth factors, such as transforming growth factor, which may promote tumor growth $[13,14]$. Furthermore, the classic mathematical model by Goldie and Coldman [15] suggests that the probability of muta-

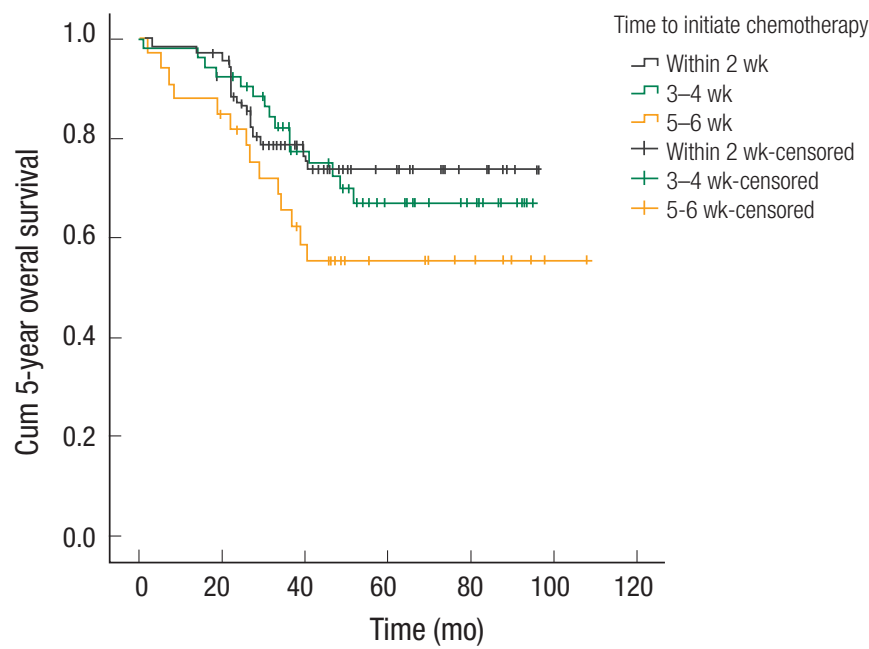

Fig. 1. Kaplan-Meier curves depicting 5-year overall survival.

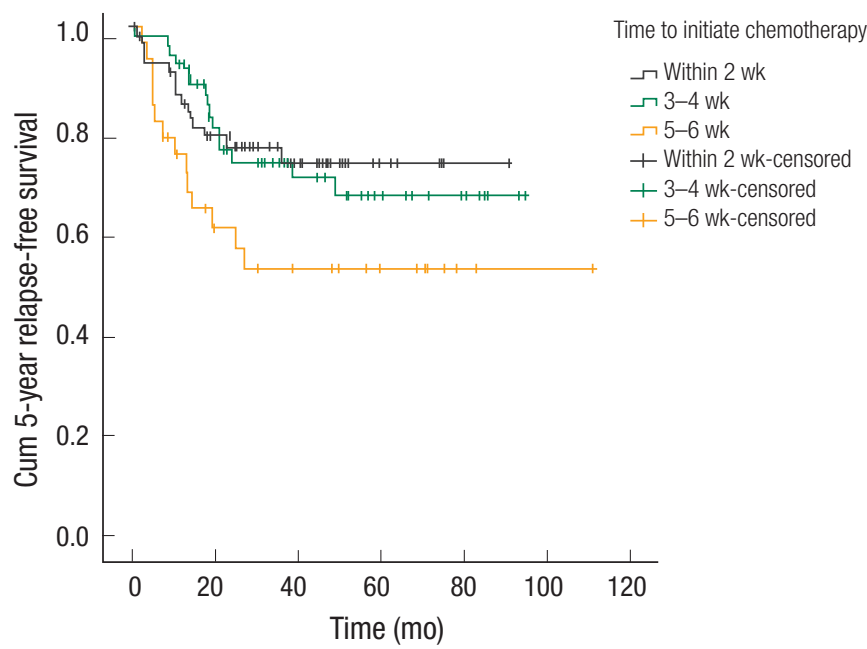

Fig. 2. Kaplan-Meier curves depicting 5-year relapse-free survival. 
tions that lead to drug resistance increases over time and depends on the mutation rate of tumor cells and their sizes. These reports of preclinical tumor growth and kinetic models support a hypothesis that chemotherapy is most effective if initiated promptly when tumor burden is low. Also, in the aspect of pharmacodynamics, the reduction of malignant proliferation is most effective when a chemotherapeutic agent is administered immediately after tumor removal. The reason behind this theory is that after surgery, the cell cycle of micrometastatic foci is very rapid due to the effect of several cytokines and this rapid cell cycle is a good target for chemotherapeutic action. The wound healing process creates a favorable environment for metastatic clones to grow, and the chemotherapeutic agent can penetrate into tumor cells more easily when the tumor burden is still small [16]. Chau et al. [17] reported that patients who started chemotherapy within 8 weeks after surgery for stage II and III CRC showed higher OS than the patients who had chemotherapy after 8 weeks, but not beyond 12 weeks. Czaykowski et al. [4] compared the OSs for stage III CRC patients for whom chemotherapy was initiated within 8 weeks of surgery and beyond 8 weeks after surgery and reported that former group showed a better result. The study results of Chau et al. [17] and Czaykowski et al. [4] supported the idea that adjuvant chemotherapy should start as soon as possible after the surgery, with most clinical trials recommending the initiation of chemotherapy within 6 or 8 weeks after surgery.

Clinical trials in CRC generally follow a time interval of no more than 6 to 8 weeks after surgery to start the adjuvant chemotherapy regimen. However, not enough prior studies have specifically examined factors associated with the time interval to initiate adjuvant chemotherapy or the relationship between the time interval and the oncologic outcome. Thus, our study analyzed the longterm follow-up results of adjuvant chemotherapy initiated within 2 weeks, within 3-4 weeks, and within 5-6 weeks after surgery, as supported by the previously-discussed hypothesis on tumor relapse. According to our analysis, group 1 had adjuvant chemotherapy within 2 weeks and had the highest 5-year OS of $73.7 \%$. group 2, which started chemotherapy within 3-4 weeks, had a 5 -year OS of $67.0 \%$. Lastly, group 3 had the lowest OS, 55.2\%. Taal et al. [18] reported results similar to those of our study; they analyzed the OSs and the RFSs of the patients for whom chemotherapy had been initiated between 20 and 28 days (between 3 and 4 weeks) and beyond 28 days (beyond 4 weeks) after surgery for stage II and III CRC. The results for each group were compared, but significant differences were found. Putting these various reports together with our study, we found that starting chemotherapy within 6 or 8 weeks after surgery had a survival benefit, but, in contrast to our expectation, no improvement of oncologic result was found to be associated with early initiation of chemotherapy.

It is important to note that initiating chemotherapy too early may cause some problems and occasionally, even death [19]. The problems include hematological, gastrointestinal, dermatological, renal, pulmonary, cardiac, neurological, hepatic and gonadal side effects. In patients with depressed immune condition and poor performance scale after surgery, the toxicity of chemotherapeutic agents may be maximized. This toxicity can affect the result of surgery and may, for example, cause postoperative bleeding due to thrombocytopenia, severe anemia and various infectious complications. Several animal studies have reported that after systemic infusion of chemotherapy, there is an increased risk of anastomotic site rupture due to the anastomosis being weaker immediately after surgery [20]. Most study results on the treatment of colon cancer point out that the benefit of starting chemotherapy earlier than 6 weeks after surgery is unclear. Our study produced the same result that initiating chemotherapy too early may not have any benefits, but may have more side effects. Thus, it would be better to wait for patients to recover and to delay chemotherapy in case of the patients with poor postoperative condition in order to lessen complications induced by chemotherapy without impairing the prognosis [19,20].

This study has some limitations. First, our study was limited by its retrospective nature. Certain imbalances of known and unknown prognostic factors, such as completeness of the regimen or the specific chemotherapy regimens received, may influence the results. In addition, some factors that might influence the decision to delay the initiation of chemotherapy and might bring about poor outcomes may not have been taken into account. Such unrecorded clinical factors that might affect overall patient survival were surgical complications, number of metastatic lymph nodes, tumor grade, and functional status of the patients. Second, the conclusions based on subgroup analyses were limited by the small numbers of patients participating in the study. Third, our study was a nonrandomized, prospective problem. For obvious ethical reasons, a randomized controlled trial comparing the survival rates for three different times to begin adjuvant chemotherapy following surgery would not be feasible. Therefore, the question of randomly having different timings for the initiation of chemotherapy cannot be addressed prospectively. If this question is to be answered, a comparison between the risk-to-benefit ratio of delayed adjuvant chemotherapy and no adjuvant chemotherapy by means of a randomized controlled trial is needed. However, such a trial would raise not only ethical problems but also practical issues. Fourth, our study does not have a comparison group for adjuvant chemotherapy. Therefore, it is impossible to speculate whether the benefit of chemotherapy is lost entirely or is partially diminished by a delay of more than 6 weeks after surgery $[16,21]$. Before conducting our study, we expected to find results that would support the use of adjuvant chemotherapy within 6 weeks after surgery to achieve a better OS, but our results did not show a significant difference depending on the timing of the initiation of adjuvant chemotherapy. Previous studies on the treatment of CRC recommended that adjuvant chemotherapy should begin 6-8 weeks after the operation, and our study result showed that beginning adjuvant chemotherapy earlier than 6 weeks did not have any significant benefits and that the general condition of the pa- 
tients should be taken into consideration when determining the optimal starting date for chemotherapy.

\section{CONFLICT OF INTEREST}

No potential conflict of interest relevant to this article was reported.

\section{REFERENCES}

1. Parkin DM, Bray F, Ferlay J, Pisani P. Global cancer statistics, 2002. CA Cancer J Clin 2005;55:74-108.

2. Jemal A, Siegel R, Xu J, Ward E. Cancer statistics, 2010. CA Cancer J Clin 2010;60:277-300.

3. Jung KW, Park S, Kong HJ, Won YJ, Lee JY, Park EC, et al. Cancer statistics in Korea: incidence, mortality, survival, and prevalence in 2008. Cancer Res Treat 2011;43:1-11.

4. Czaykowski PM, Gill S, Kennecke HF, Gordon VL, Turner D. Adjuvant chemotherapy for stage III colon cancer: does timing matter? Dis Colon Rectum 2011;54:1082-9.

5. Bayraktar S, Bayraktar UD, Rocha-Lima CM. Timing of adjuvant and neoadjuvant therapy in colorectal cancers. Clin Colorectal Cancer 2010;9:144-9.

6. Cheung WY, Neville BA, Earle CC. Etiology of delays in the initiation of adjuvant chemotherapy and their impact on outcomes for Stage II and III rectal cancer. Dis Colon Rectum 2009;52:1054-63.

7. Lima IS, Yasui Y, Scarfe A, Winget M. Association between receipt and timing of adjuvant chemotherapy and survival for patients with stage III colon cancer in Alberta, Canada. Cancer 2011;117: 3833-40.

8. Biagi JJ, Raphael MJ, Mackillop WJ, Kong W, King WD, Booth $\mathrm{CM}$. Association between time to initiation of adjuvant chemotherapy and survival in colorectal cancer: a systematic review and meta-analysis. JAMA 2011;305:2335-42.

9. McCulloch P, Choy A. Effect of menstrual phase on surgical treatment of breast cancer. Lancet 1994;344:402-3.

10. Fidler IJ, Ellis LM. The implications of angiogenesis for the biolo- gy and therapy of cancer metastasis. Cell 1994;79:185-8.

11. Folkman J. What is the evidence that tumors are angiogenesis dependent? J Natl Cancer Inst 1990;82:4-6.

12. Gunduz N, Fisher B, Saffer EA. Effect of surgical removal on the growth and kinetics of residual tumor. Cancer Res 1979;39:38615.

13. Ono I, Gunji H, Suda K, Iwatsuki K, Kaneko F. Evaluation of cytokines in donor site wound fluids. Scand J Plast Reconstr Surg Hand Surg 1994;28:269-73.

14. Eggermont AM, Steller EP, Sugarbaker PH. Laparotomy enhances intraperitoneal tumor growth and abrogates the antitumor effects of interleukin-2 and lymphokine-activated killer cells. Surgery 1987;102:71-8.

15. Goldie JH, Coldman AJ. A mathematic model for relating the drug sensitivity of tumors to their spontaneous mutation rate. Cancer Treat Rep 1979;63:1727-33.

16. Des Guetz G, Nicolas P, Perret GY, Morere JF, Uzzan B. Does delaying adjuvant chemotherapy after curative surgery for colorectal cancer impair survival? A meta-analysis. Eur J Cancer 2010;46: 1049-55.

17. Chau I, Norman AR, Cunningham D, Tait D, Ross PJ, Iveson T, et al. A randomised comparison between 6 months of bolus fluorouracil/leucovorin and 12 weeks of protracted venous infusion fluorouracil as adjuvant treatment in colorectal cancer. Ann Oncol 2005;16:549-57.

18. Taal BG, Van Tinteren H, Zoetmulder FA; NACCP group. Adjuvant 5FU plus levamisole in colonic or rectal cancer: improved survival in stage II and III. Br J Cancer 2001;85:1437-43.

19. Goldman LI, Lowe S, al-Saleem T. Effect of fluorouracil on intestinal anastomoses in the rat. Arch Surg 1969;98:303-4.

20. Morris T. Retardation of healing of large-bowel anastomoses by 5-fluorouracil. Aust N Z J Surg 1979;49:743-5.

21. Lohrisch C, Paltiel C, Gelmon K, Speers C, Taylor S, Barnett J, et al. Impact on survival of time from definitive surgery to initiation of adjuvant chemotherapy for early-stage breast cancer. J Clin Oncol 2006;24:4888-94. 